\title{
Automatic leaking carrier canceller adjustment techniques
}

\author{
Gregor Lasser $^{1 *}$, Robert Langwieser ${ }^{2}$ and Christoph F Mecklenbräuker ${ }^{2}$
}

\begin{abstract}
In this contribution, four automatic adjustment algorithms for leakage carrier cancellation in radio frequency identification (RFID) readers are compared: full search, gradient search, fast and direct I/Q algorithms. Further, we propose two enhanced adjustment procedures.

First, we analytically calculate the performance of the fast adjustment algorithm in the presence of noise and derive its theoretical bias. We compare the theoretical results with the numerical results from accompanying simulations. Further, we evaluate the performance of these algorithms based on real-world measurements acquired with our RFID testbed.

Finally, we propose and discuss the merits of two enhanced adjustment procedures based on the fast adjustment algorithm. The fast adjustment procedure with bipolar probing signals achieves the isolation gain of the (much slower) gradient search algorithm at the expense of a mean penalty of $0.48 \mathrm{~dB}$. We observe that the fast adjustment aided gradient algorithm requires $72 \%$ less steps than the gradient search algorithm in our measurements.
\end{abstract}

\section{Introduction}

Radio frequency identification (RFID) is a technique to remotely identify and detect objects that are branded with a special transponder called RFID tag [1]. RFID systems operate at several frequency bands and use different methods to transfer data and energy between an RFID reader and the tags. In this work, we will focus on RFID systems that use electromagnetic waves for communications, especially ultrahigh frequency (UHF) RFID systems.

An RFID tag consists of an antenna that is connected to an electronic circuit, which in most cases is built on an integrated circuit. Many RFID systems use the so-called passive or semi-passive tags that do not use an internal power source to communicate with the RFID reader. They instead use backscattering, a technique which is based on the fact that the amplitude and phase of the waves scattered from an antenna depend on the antenna termination impedance. Thus, the tag sends data to the reader by modulating the impedance that the tag chip presents to the antenna terminals $[2,3]$. While this backscattering technique, seen from the tag, enables remotely powered

\footnotetext{
*Correspondence: gregor.lasser@nt.tuwien.ac.at

1 Institute of Telecommunication, Vienna University of Technology,

1040,Vienna, Austria

Full list of author information is available at the end of the article
}

communication, it necessitates a constant carrier signal to be transmitted from the RFID reader during the tag to reader data transfer [4-6]. Therefore, the RFID reader has to transmit a carrier signal while it simultaneously receives a weak backscattered signal from the tag. To separate transmit an receive paths, readers either use separate transmit and receive antennas or use circulators or directional couplers. In analogy to radar systems, the first case is called bistatic, while the second one is called monostatic. Still, both system concepts struggle with low transmitter to receiver isolations [7]. This demands for receivers with very large dynamic ranges, which enhance costs, both on the analog front end as well as on the ana$\log$ to digital converters. To reduce these demands, many authors [6,8-17] as well as commercial monolithic RFID reader chip manufacturers $[18,19]$ propose or use active leakage cancellation techniques. These techniques, which originate in radar [20,21], extract a part of the transmit signal, adjust it in amplitude and phase and inject it at the receiver. When the amplitude is adjusted to be equal and the phase to be opposite of the leakage signal, the deliberately added signal and the leakage signal cancel. While there exist many publications on hardware implementations of these leaking carrier cancellers (LCCs), few exist

\section{是 Springer}

(c) 2013 Lasser et al: licensee Springer. This is an Open Access article distributed under the terms of the Creative Commons Attribution License (http://creativecommons.org/licenses/by/2.0), which permits unrestricted use, distribution, and reproduction in any medium, provided the original work is properly cited. 
on adjustment algorithms to adapt them. However, adaption is critical $[22,23]$ because typical RFID scenarios like warehouses and conveyor belts change permanently and therefore cannot be adjusted statically.

In this paper, we will accomplish the following:

- Present a comparison of four adjustment algorithms regarding their demands on hardware linearity, detector type and LCC calibration, and their setting speed,

- Analytically and numerically analyse the noise performance of the fast algorithm including a bias derivation,

- Practically compare the fast algorithm with the gradient search algorithm using our RFID testbed,

- Present an enhancement to the fast algorithm, which both gives better results under nonlinear detector conditions as well as a better noise performance,

- Report on observed step number reductions using the result of the fast algorithm for initialising the gradient algorithm: $72 \%$ reduction on average in our experiment.

The paper is structured as follows: In Section 2, we will describe the principals of leakage cancellation based on a generic RFID reader model. We will then present four automatic LCC adjustment routines and compare them regarding hardware demands and speed. This will be discussed in the context of detector types and positions with regard to our generic reader. In Section 3, we will provide a noise analysis for the fast algorithm which includes analytic and numeric results. Measurements on our RFID testbed will be described in Section 4. Finally, we will present two enhanced adjustment procedures based on the practical findings of the measurements, and we will compare them with the algorithms described before.

\section{LCC adjustment principles}

In this section, we explain the principal possibilities of active leakage cancellation adjustment techniques. Figure 1 illustrates a simplified block diagram of a bistatic RFID reader. The upper part of the figure shows the transmitter, which is composed of a signal generator, a modulator to send data to the tag, a power amplifier to create the necessary output power and a directional coupler. This coupler is used to extract a small part of the transmit signal and feed it into the LCC. Most of the transmit power is fed to the transmit antenna, where it is radiated. The lower part of Figure 1 illustrates the reader's receiver part. The receive antenna picks up the tag's response and an unwelcome leakage signal from the transmitter. Both signals path a band pass filter and directional couplers. The first coupler enables injection of the cancellation signal from the LCC. The second coupler feeds detector
(Det.) A. The coupler is followed by a low-noise amplifier (LNA) and a mixer stage. The received signal is filtered and amplified by a variable gain amplifier. Here, again a directional coupler is present to feed Det. B. The coupler is followed by an analog to digital converter (ADC), which converts the received signal into a data stream. Detector $C$ is implemented in the digital domain.

The LCC consists of a vector modulator and an amplifier. The vector modulator is controlled by the reader control block and enables to adjust the amplitude and phase of the transmit signal sample to cancel the leakage signal in the receiver's directional coupler. The amplifier compensates for the coupling losses and enables cancellation of strong leakage signals.

Conventional RFID readers rarely have that many detectors as were described before. However, at least one is necessary to implement any LCC adjustment routine, but not every detector position or type supports every algorithm. We use the very general set-up described in Figure 1 to exemplify RFID reader implementations with focus on possible LCC control implementations. Besides the location, we distinguish between scalar power detectors and vector detectors which also capture the phase of the incoming signal. The second type requires a reference signal that is either supplied from the reader's transmitter part or from the ADC sampling clock. We use this generic RFID reader model through the next sections where we describe different automatic LCC adaption techniques, which aim to find the optimum inphase $(I)$ and quadrature $(Q)$ component settings for the LCC.

Our generic model is applicable to stationary RFID readers as well as to handheld devices. The latter usually employ integrated antennas which enable a better control of the typical expected leakage values and potentially a simpler LCC design. To save space and costs, the LCC may also be included in the antenna which was presented in [16]. Besides these differences, all LCC circuits need to be adjusted. While it might be desirable for mobile devices to reduce hardware complexity for smaller packages and lower costs, we will see that there is a tradeoff between adjustment speed and necessary hardware complexity for the different adjustment algorithms. Since handheld readers most likely are moved all the time during their use, permanent and fast adjustment routines are beneficial.

\subsection{Full search algorithm}

The most primitive way to find the optimum setting for an LCC is by trying all possible LCC settings and picking the one which proved to have the best result. If inphase and quadrature components both have $N$ settings, $N^{2}$ measurements have to be performed. Thus, one obvious drawback of this technique is the large amount of measurements and adjustment steps which need to be 


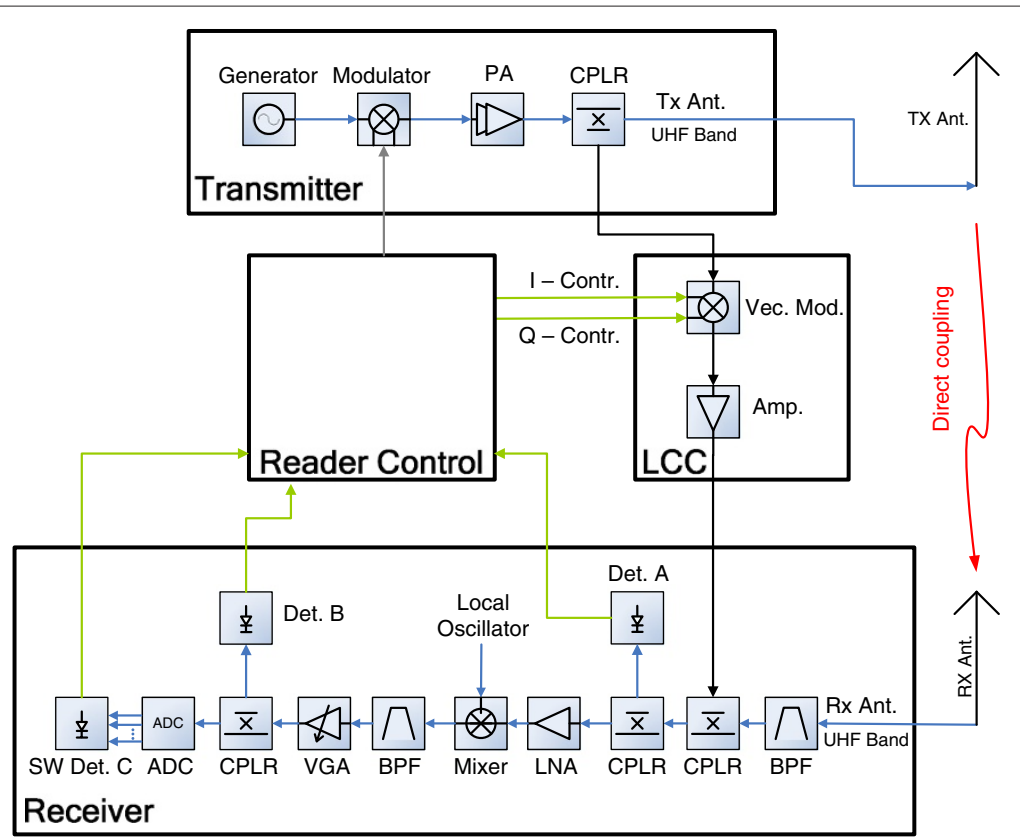

Figure 1 Block diagram of an RFID reader. Block diagram of a simplified RFID reader showing possible detector positions for LCC adjustment routines. PA, power amplifier; CPLR, coupler; I, inphase; Q, quadrature; Det., detector; ADC, analog to digital converter; VGA, variable gain amplifier; BPF, band pass filter; LNA, low-noise amplifier; TX Ant., transmit antenna; RX Ant., receive antenna; UHF, ultrahigh frequency; Vec. Mod, vector modulator; SW, software.

performend before the final result is gained. Depending on the speed of the given detector hardware, this corresponds to a large overall scanning time of the LCC which might be inadequate even for moderately changing environments. In these cases, this algorithm may completely fail to find an LCC setting because the slowly moving optimum LCC setting might never be hit during the scanning process. Besides these obvious disadvantages, the full search algorithm has the benefit of accepting any type of power detector as long as it shows a monotone, but not necessary linear input-output relation. Here, we mean monotone if the output of the detector is increasing (or remains constant) when the input power is increasing. Even if the receiver is completely overloaded by the leakage signal for most LCC settings, the power detector will provide the lowest output signal at the appropriate LCC setting. Therefore, this algorithm may be used in receiver structures, which do not employ a special power detector for LCC adjustment, and it has very limited demands on receiver linearity. For these reasons, some commercial reader chips use this algorithm [18]. An improvement to reduce the scanning time, which is also implemented in [18], is to divide the algorithm into two steps and to scan only a fraction of all $N^{2}$ setting in the first step. When this subgrid is properly chosen, at least one of the scanned subgrid points is close to the appropriate LCC setting, and therefore, the receiver operates in the linear regime and gives useful power detector readings. In the second step, only the vicinity of the point with the lowest measured remaining LCC power is scanned.

\subsection{Gradient search algorithm}

The gradient search algorithm, which is also called method of the steepest descent ([24], Sec. 5.3), is an iterative approach to find the optimum LCC setting. For every step $n$, the algorithm finds the local gradient $\nabla_{n}$ of the power distribution versus the LCC setting. This is done by performing three measurements: First, the power at the actual LCC setting $c_{n}$ is recorded. Then, the LCC setting is changed for some small value $\delta$ in the inphase component only, and again, the resulting power is recorded. The last measurement records the power after applying a small change in the LCC's quadrature component only. Based on these three power values, the local gradient to the power distribution $\nabla_{n}$ is calculated, and a new LCC setting is found by the following:

$$
c_{n+1}=c_{n}-\mu \nabla_{n},
$$

where $\mu$ is a positive, real-valued constant called the stepsize parameter. Beginning from a starting point that is usually set to zero, $c_{0}=0$, the gradient search algorithm step by step tries to approach the optimum LCC setting. Setting the step-size parameter $\mu$ is critical for this algorithm to work: If it is too small, many steps are necessary to obtain the final value. Setting it too high results in oscillations, and the algorithm will not converge. A similar 
problem exists for the deviation value $\delta$ that is used to measure the gradient. Choosing $\delta$ too large will possibly lead to false gradient measurements when it is applied at close proximity to the optimum value. A small $\delta$ implies a small change in power and in the presence of noise an unreliable gradient measure.

Since it is an iterative technique, the gradient search algorithm is well suited for changing environments. Another benefit of this technique are the moderate linearity constraints which it imposes on the reader. As long as the power detector is strictly monotone and the step width is chosen small enough to ensure stability, the algorithm will converge to the optimum LCC setting point. Few authors explicitly state adjustment algorithms, but the authors in [16] are using a gradient-based approach as well as in [12], who describe a similar approach and use a variable step width.

\subsection{Fast algorithm}

The fast algorithm is a new technique which was first published in [25]. In this section, we will give a short description of the principle operation, while in later sections, a noise analysis, numerical simulations and measurement results are presented.

The algorithm gains the optimum LCC setting by obtaining three amplitude measurements. These measurements are very similar to the ones performed to gain the gradient in the gradient algorithm, but in contrast to the gradient algorithm, they do not provide an enhancement in information for the next iteration but immediately provide the correct LCC setting.

In the first step, the plain leakage amplitude without any LCC signal is measured by setting the complex LCC output $c_{0}=0$ and measuring the input amplitude:

$$
r_{0}=\sqrt{x^{2}+y^{2}},
$$

where $x$ and $y$ are the unknown $I$ and $Q$ components of the leakage signal.

In the second step, we apply a signal of amplitude $r_{0}$ with the $I$ channel of the LCC. Thus, the complex output signal of the LCC is $c_{1}=r_{0}+j 0$. The amplitude measurement in this step equals

$$
r_{1}=\sqrt{\left(x+r_{0}\right)^{2}+y^{2}} .
$$

In the third step, we now probe the quadrature component of the leakage by setting the LCC to $c_{2}=j r_{0}$ and receive

$$
r_{2}=\sqrt{x^{2}+\left(y+r_{0}\right)^{2}} .
$$

With these measurements, we find the components of the leaking signal by calculating the following:

$$
\begin{aligned}
& \hat{x}=\frac{\frac{1}{2} r_{1}^{2}-r_{0}^{2}}{r_{0}}=x, \\
& \hat{y}=\frac{\frac{1}{2} r_{2}^{2}-r_{0}^{2}}{r_{0}}=y .
\end{aligned}
$$

Thus, the optimum LCC setting is found to be $c_{\mathrm{opt}}=$ $-x-j y$.

In contrast to the techniques described before, the detector has to be linear in amplitude for this algorithm to perform satisfactorily. The benefit of this approach is that it is very fast and may be used for changing environments as long as the leakage channel remains constant during the three measurement steps, which is the same condition as for the gradient algorithm. However, the fast algorithm requires calibration of the LCC settings, which means that for a pure LCC signal the relation between detector readings and LCC setting has to be known. A practical implementation of this calibration is explained in Section 4.1. Based on the calibration, it is possible to generate the appropriate probing signal amplitudes and finally the compensation signal $c_{\mathrm{opt}}$ based on Equation 5 .

\subsection{Direct $I / Q$ algorithm}

This is a straightforward technique which requires a receiver equipped with a vector signal detector. Additionally, the LCC settings have to be calibrated with respect to this detector. Once a leakage signal is received, the inphase and quadrature components of this leakage signal are detected in the vector detector. The only necessary step is to set the LCC $I$ and $Q$ values opposite to the leakage signal. The benefit of this approach is extreme speed, when compared to all other techniques. However, it is the technique with the highest hardware demands, both for the detector type and the necessary LCC calibration.

The vector detector may either be implemented in the digital domain as detector $\mathrm{C}$ in Figure 1 or as a separate hardware vector detector in positions A or B. The authors of [10] present a receiver structure which employs a dedicated hardware receiver in position A which is suitable for a direct $I / Q$ detection algorithm.

\subsection{Comparison of adjustment principles}

Before we start our description of detector positions and their influence on detector performance, we summarise the descriptions of the described algorithms in Table 1. The algorithm name is given in the first column; the second column states the detector type which is employed. While the full search and gradient search algorithms only require power values, the fast algorithm commands for amplitude values, and the direct $I / Q$ algorithm even 
Table 1 Comparison of LCC adjustment techniques

\begin{tabular}{llccc}
\hline Algorithm & Detector type & Linearity constraint & Number of steps & LCC calibration \\
\hline Full search & Power & None & $N^{2}$ & No \\
Gradient search & Power & Low & 3 to $\frac{3 N}{2}$ & Minimal \\
Fast algorithm & Amplitude & High & 3 & Yes \\
Direct I/Q & I/Q amplitude & High & 1 & Yes \\
\hline
\end{tabular}

This table compares four LCC adjustment algorithms with respect to hardware demands and number of steps, where every setting of the LCC counts as one step. The number of LCC settings in I and $Q$ domains is assumed to be identical and equal to $N$.

demands for a vector amplitude, i.e. an $I / Q$ amplitude detector. The third column lists the linearity constraints of the algorithms, and the fourth column states the number of steps which every algorithm requires to find $c_{\text {opt }}$. Here, we define the step as the process of setting the LCC to a certain value and taking a measurement. The full search algorithm scans the complete $I / Q$ plane of the LCC, so it takes $N^{2}$ steps when $N$ is the number of $I$ and $Q$ settings. As the gradient algorithm is iterative, the necessary number of iterations is unknown. Every iteration requires three measurements; therefore, the minimum number of steps is equal to three. When the algorithm converges without oscillations, the maximum number of iterations is half of the $I$ and $Q$ plane width, corresponding to $\frac{3 N}{2}$ steps. The fast algorithm takes three measurements which corresponds to three steps in Table 1. As was described before, the full search and the gradient search do not require any $\mathrm{LCC}$ calibration, while the fast algorithm and the direct $I / Q$ method do. However, for picking the proper step width $\mu$, the maximum change per LCC step needs to be known which corresponds to the 'minimal' entry in Table 1.

\subsection{Detector positions}

We now discuss the possible detector positions with respect to our principal RFID reader described in Figure 1. Detector $\mathrm{A}$ is positioned right at the beginning of the receiver. No active components precede this detector, so only the detector itself is defining its output linearity. Therefore, this position is well suited for algorithms which require a linear detector behaviour like the fast algorithm or the direct $I / Q$ method. However, for the second, a more complex vector detector needs to be implemented. The other two algorithms will also operate properly with this detector.

Detector position B is positioned at the end of the analog receiver chain either at a low intermediate frequency or at the baseband. When compared to detector A, higher signal levels and lower frequencies are present at position $\mathrm{B}$, and therefore, the implementation of the detector itself is less demanding. However, the receiver chain which precedes the detector might degrade the linearity of the detector. Therefore this position is well suited for techniques with low linearity constraint, like the full search algorithm or the gradient search approach.

Detector $C$ in general does not require any additional hardware because it is implemented in a software. It is relatively easily implemented as a vector power detector. The drawback of this detector is the fact that the complete receiver chain including amplifiers, mixers and ADCs is passed before the detector. For linear functionality, this complete chain has to operate in the linear domain as well. This increases the requirements on the whole receiver and at the end makes the use of an LCC questionable if the receiver operates in a linear fashion under leakage carrier conditions, why bother to implement an LCC? It still makes sense to compensate the leakage in this case because the requirements on dynamic range regarding detecting the weak received tag response under the presence of leakage are very demanding, especially when we concern the necessary ADC resolution.

\section{Noise analysis}

In this section, we will present a detailed noise analysis for the fast algorithm. We will derive the estimator's bias and error variance as a function of the carrier to noise ratio (CNR). For the other algorithms, a short description follows.

As the full search algorithm searches the lattice of $N^{2}$ LCC setting points and picks the best one, the final setting error is not only constraint on the CNR at the detector but also on the quantisation error due to the finite number of setting points. For high CNR values, the correct point will be picked with high probability, and the setting error is dominated by the quantisation error which is proportional to $\frac{1}{N^{2}}$ ([26], Chap. 5.6). For the low CNR regime or when $N$ is very large, noise limits the detection of the minimum power at the optimum LCC setting point when scanning the complete $I / Q$ plane. Since this null is rather distinct $[15,17]$, the noise influence is low.

For the gradient search algorithm, the noise influence is uncritical due to its iterative nature. This is of course only true if the step size is chosen small enough to guarantee convergence under noise influence.

The noise analysis for the direct $I / Q$ algorithm is straightforward and we will use it as a reference for the fast 
algorithm. Since the $I$ and $Q$ components of the leakage signal are directly measured by an appropriate detector, the detector noise variance and bias are equal to the algorithm's error noise variance and bias.

\subsection{Analytic analysis}

In this section, we expand the noise free description of the fast algorithm given in Section 2.3 for the case which includes noisy $I$ and $Q$ components. In an actual RFID reader implementation, many parts in the transmitter, channel, receiver, detector and possibly an ADC following the detector contribute to noise which degrades the measurements described in Equations 2 to 4. Depending on the underlying physics of these noise sources and their position in the TX-RX chain, their noise has to be modelled in different ways. In the following section, we will focus on strictly white, statistically independent noise that adds to the $I$ and $Q$ components. Further, we focus on the estimation of the inphase component $\hat{x}$, as these results later may easily be adapted to $\hat{y}$.

To include the noise, we replace the noise-free leakage components $x$ and $y$ with the following:

$$
x_{i}=x+u_{i}, \quad y_{i}=y+v_{i},
$$

where $u_{i}$ and $v_{i}$ are the realisations of the statistically independent noise processes $U$ and $V$, respectively. We expand Equation 2 and get the following:

$$
r_{0}=\sqrt{x_{0}^{2}+y_{0}^{2}}=\sqrt{\left(x+u_{0}\right)^{2}+\left(y+v_{0}\right)^{2}},
$$

which includes the noise realisations $u_{0}$ and $v_{0}$ of the first step. We apply noise to the second measurement as well and receive

$$
r_{1}=\sqrt{\left(x_{1}+r_{0}\right)^{2}+y_{1}^{2}}=\sqrt{\left(x+u_{1}+r_{0}\right)^{2}+\left(y+v_{1}\right)^{2}} .
$$

We proceed to calculate the expectation of the estimated I component $\hat{x}$ :

$$
\begin{aligned}
E\{\hat{x}\}= & E\left\{\frac{\frac{1}{2} r_{1}^{2}-r_{0}^{2}}{r_{0}}\right\}=E\{x\}+E\left\{u_{1}\right\} \\
& +E\{\underbrace{\frac{x u_{1}+y v_{1}+\frac{u_{1}^{2}+v_{1}^{2}}{2}}{r_{0}}}_{E_{1}}\} \\
& -E\{\underbrace{\frac{x u_{0}+y v_{0}+\frac{u_{0}^{2}+v_{0}^{2}}{2}}{r_{0}}}_{E_{2}}\} \\
= & x+\mu_{u}+E\left\{E_{1}\right\}-E\left\{E_{2}\right\} .
\end{aligned}
$$

This expectation includes $x$ and an additive bias term. To treat the bias, we specialise our noise model: The noise of both signal components $U$ and $V$ is white and Gaussian and has zero mean $\mu_{u}=\mu_{v}=0$. The noise variances are $\sigma_{u}^{2}$ and $\sigma_{v}^{2}$. As stated before, we presume statistical independence of the $I$ and $Q$ noise components.

The first bias term $E_{1}$ can be divided in the expectations of the numerator and $1 / r_{0}$ since the numerator and denominator are statistically independent. Therefore, we compute the expectation of $E_{1}$ as follows:

$$
\begin{aligned}
E\left\{E_{1}\right\}= & E\left\{x u_{1}+y v_{1}+\frac{u_{1}^{2}+v_{1}^{2}}{2}\right\} E\left\{\frac{1}{r_{0}}\right\}=\frac{\sigma_{u}^{2}+\sigma_{v}^{2}}{2} \\
& \times E\left\{\frac{1}{\sqrt{x^{2}+y^{2}+2 x u_{0}+2 y v_{0}+u_{0}^{2}+v_{0}^{2}}}\right\} \\
= & \frac{\sigma_{u}^{2}+\sigma_{v}^{2}}{2 r} E\left\{\frac{1}{\sqrt{1+\frac{2 x u_{0}+2 y v_{0}}{r^{2}}+\frac{u_{0}^{2}+v_{0}^{2}}{r^{2}}}}\right\} \\
= & \frac{\sigma_{u}^{2}+\sigma_{v}^{2}}{2 r} E\left\{\frac{1}{\sqrt{1+\varepsilon}}\right\},
\end{aligned}
$$

where $r=\sqrt{x^{2}+y^{2}}$ is the noise-free amplitude of the leakage signal. To compute the result of the remaining expectation operator, which acts as a weighting factor for the preceding term including the noise variances, we approximate the root expression by a first-order Taylor expansion for $\varepsilon$. This approximation holds when the noise components are small when compared to the carrier power $r^{2}$, i.e. the CNR is high.

$$
\begin{aligned}
E\left\{E_{1}\right\} \approx & \frac{\sigma_{u}^{2}+\sigma_{v}^{2}}{2 r} E\left\{1-\frac{\varepsilon}{2}\right\}=\frac{\sigma_{u}^{2}+\sigma_{v}^{2}}{2 r} \\
& \times E\left\{1-\frac{x u_{0}+y v_{0}}{r^{2}}-\frac{u_{0}^{2}+v_{0}^{2}}{2 r^{2}}\right\} \\
= & \frac{\sigma_{u}^{2}+\sigma_{v}^{2}}{2 r}-\frac{\sigma_{u}^{4}+2 \sigma_{u}^{2} \sigma_{v}^{2}+\sigma_{v}^{4}}{4 r^{3}} .
\end{aligned}
$$

We treat the second bias term $E_{2}$ in a different way, since here the separation of numerator and denominator is not possible. Instead, we reformulate the following problem: 


$$
\begin{aligned}
& E_{2}=\frac{x u_{0}+y v_{0}+\frac{u_{0}^{2}+v_{0}^{2}}{2}}{r \sqrt{1+\frac{2 x u_{0}+2 y v_{0}}{r^{2}}+\frac{u_{0}^{2}+v_{0}^{2}}{r^{2}}}} \\
& =\frac{r\left(-1+1+\frac{2 x u_{0}+2 y v_{0}}{r^{2}}+\frac{u_{0}^{2}+v_{0}^{2}}{r^{2}}\right)}{2 \sqrt{1+\underbrace{\frac{2 x u_{0}+2 y v_{0}}{r^{2}}+\frac{u_{0}^{2}+v_{0}^{2}}{r^{2}}}_{\varepsilon}}} \\
& =\frac{r}{2}\left(\sqrt{1+\varepsilon}-\frac{1}{\sqrt{1+\varepsilon}}\right) .
\end{aligned}
$$

Similar to the step we used before, we approximate the expression in the brackets using a second-order Taylor expansion. We compute the expectation as follows:

$$
\begin{aligned}
E\left\{E_{2}\right\} & \approx E\left\{\frac{r}{2}\left(\varepsilon-\frac{\varepsilon^{2}}{2}\right)\right\} \\
& =r\left(\frac{\sigma_{u}^{2}+\sigma_{v}^{2}}{2 r^{2}}-\frac{x^{2} \sigma_{u}^{2}+y^{2} \sigma_{v}^{2}}{r^{4}}-\frac{3 \sigma_{u}^{4}+2 \sigma_{u}^{2} \sigma_{v}^{2}+3 \sigma_{v}^{4}}{4 r^{4}}\right),
\end{aligned}
$$

using the fact that $E\left\{u_{0}^{4}\right\}=3 \sigma_{u}^{2}$ and $E\left\{v_{0}^{4}\right\}=3 \sigma_{v}^{2}$, since $U$ and $V$ are Gaussian variables. Finally, we compute $E\{\hat{x}\}$ as follows:

$$
\begin{aligned}
E\{\hat{x}\} & =x+E\left\{E_{1}\right\}-E\left\{E_{2}\right\} \\
& \approx x+r\left(\frac{x^{2} \sigma_{u}^{2}+y^{2} \sigma_{v}^{2}}{r^{4}}+\frac{\sigma_{u}^{4}+\sigma_{v}^{4}}{2 r^{4}}\right) .
\end{aligned}
$$

For the case of equal noise variances of the $I$ and $Q$ components, we further simplify this expression using $\sigma_{u}^{2}=$ $\sigma_{v}^{2}=\sigma^{2} / 2$, where $\sigma^{2}$ denotes the variance of the circularly symmetrical complex Gaussian variable composed of $U+j V$. Using this variance, the CNR is defined as the ratio of the leaking carrier power and the complex noise variance:

$$
\begin{aligned}
E\{\hat{x}\} \approx x+r\left(\frac{\sigma^{2}}{2 r^{2}}+\frac{\sigma^{4}}{4 r^{4}}\right) & =x+r\left(\frac{1}{2 \mathrm{CNR}}+\frac{1}{4 \mathrm{CNR}^{2}}\right), \\
\text { with } \mathrm{CNR} & =\frac{r^{2}}{\sigma^{2}} .
\end{aligned}
$$

We see that the proposed fast algorithm acts as a biased estimator. Since the bias is approximately known, it can be compensated if the CNR is known as well. In most RFID applications, leakage carrier compensation is performed to reduce a large leakage signal, so high CNRs are expected. In this case, the bias may be neglected.

\subsection{Monte Carlo simulations}

We performed a Monte Carlo simulation of the fast algorithm using the same circularly symmetric complex Gaussian noise model as described before. For every CNR value, $4 \times 10^{6}$ simulations were performed. Figure 2 shows a comparison between the simulated average bias and the analytic approximate bias of Equation 15 derived before. We see that for CNRs larger than $3 \mathrm{~dB}$, both curves match very closely, and the bias of the estimator is well described in Equation 15. For lower CNR values, the approximations made in Equations 11 and 13 do not hold any more, and the curves start to diverge. This means that the bias of the estimator can be removed if the CNR is known. However, we will see that there is a good reason not to aim for an unbiased estimator for this particular problem.

Figure 3 shows the standard deviation of the standardised error $\frac{\hat{x}-x}{r}$ of the estimation of the $I$ component $x$. This is compared to the standard deviation of the standardised noise on the $I$ component, which is computed to $\frac{\sigma_{u}}{r}=\sqrt{\frac{1}{2 \mathrm{CNR}}}$. For low CNRs, the error variance is severely increased when compared to the noise variance. However, in the higher CNR regime, the difference is not so large, and both curves tend to zero. This comparison may also be interpreted as performance comparison between the fast algorithm and the direct $I / Q$ algorithm, since the latter algorithm's noise performance is directly given by the noise on each component. Thus, it is evident that the direct vector measurement of the leakage employed by the direct $I / Q$ algorithm does not show the noise enhancement and is superior for low CNRs. The third curve of the enhanced fast algorithm is discussed in Section 5.1.

Up to now, we just discussed the moments of the estimator based on the fast algorithm. Based on the Monte Carlo simulations, we now discuss scatter plots and histograms

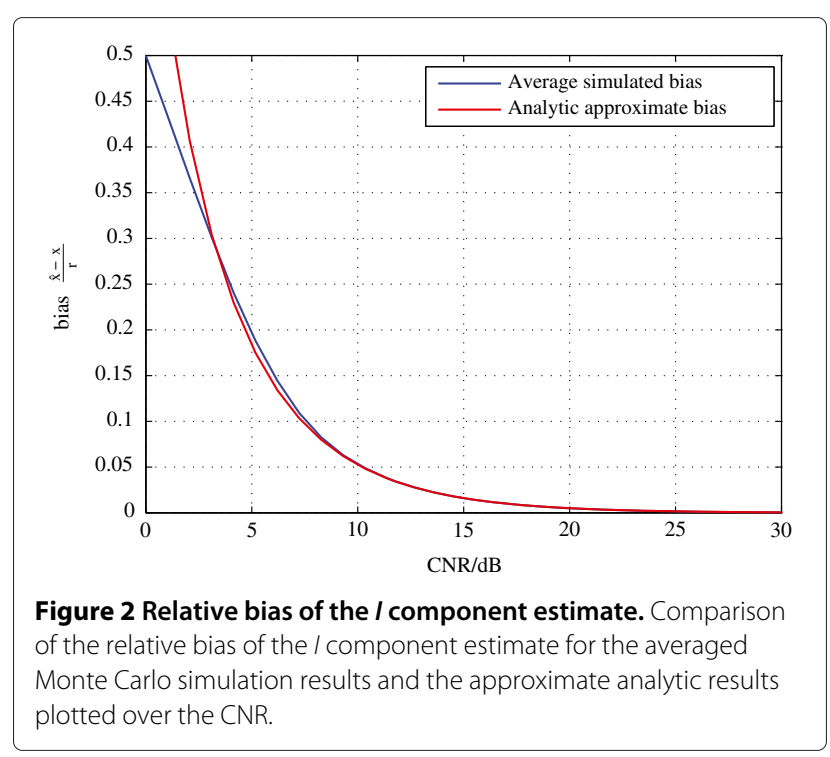




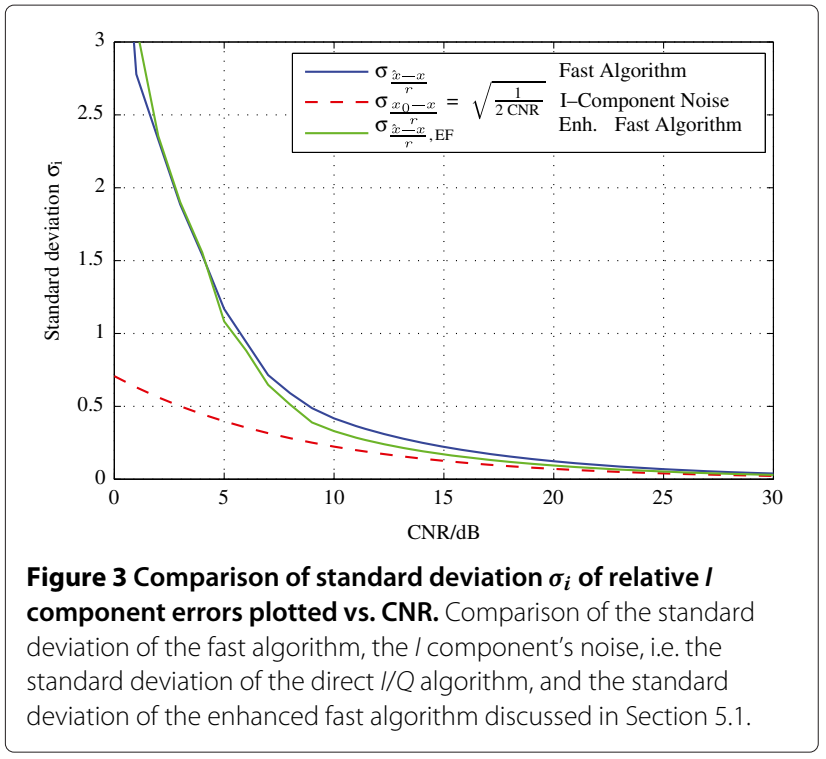

to analyse the statistical distribution of the estimation error. Figure 4a illustrates the error distribution for a very low $\mathrm{CNR}$ of $\mathrm{CNR}=-5 \mathrm{~dB}$ by showing 8,000 error points from the Monte Carlo simulations. We see that the noise is centered around the origin but, additionally, is quite spread in the sector of positive $I$ and $Q$ components. The diagram also shows the mean of the error, which was obtained by $4 \times 10^{5}$ simulations and which is at $0.87+0.87 j$. Applying a bias correction following Equation 15 leads to catastrophic overcompensation since the bias is not well captured at low CNRs as we discussed before. Further, from Figure 4a, we see that there are good reasons not even to try to compensate for the bias and use the slightly biased estimator as it is: The mode of the error, which is the peak of the histogram of the error, is slightly negative $(-0.5+-0.4 j)$, and therefore, most of the estimates of $x$ and $y$ will be slightly negatively biased. This is also clear from the histogram shown in Figure 4b. The distribution has a long tail towards positive values which shifts the mean to this side. The unbiased estimator is found by shifting this distribution to negative values, but this of course will shift the negative mode even further. Therefore, we suggest not to apply any bias correction.

The situation gets less distinct for a CNR of $0 \mathrm{~dB}$ as it is used in Figure 5. Here, the bias compensation still leads to overcompensation, but at least, the magnitude of the remaining bias is smaller. Since the mode of the error is still slightly negative for both components, the bias compensation can be omitted in favor of the closer error mode.

We see from the histogram in Figure $6 \mathrm{~b}$ that for medium to high CNRs, the error distribution becomes not only more compact, but also the long tail towards positive values is shortening. The same is observed from the scatter plot (Figure 6b), and both mean error and error mode tend to the origin. Since the whole issue of carrier cancellation is tackled to combat strong leakage signals, we expect that most RFID readers will operate in high CNR regimes so that the skewness of the fast algorithm's error distribution is not an issue, and bias compensation is superfluous.

\section{Measurements}

We performed measurements to compare the fast algorithm with the gradient algorithm. These measurements were based on our RFID testbed described in [27], which
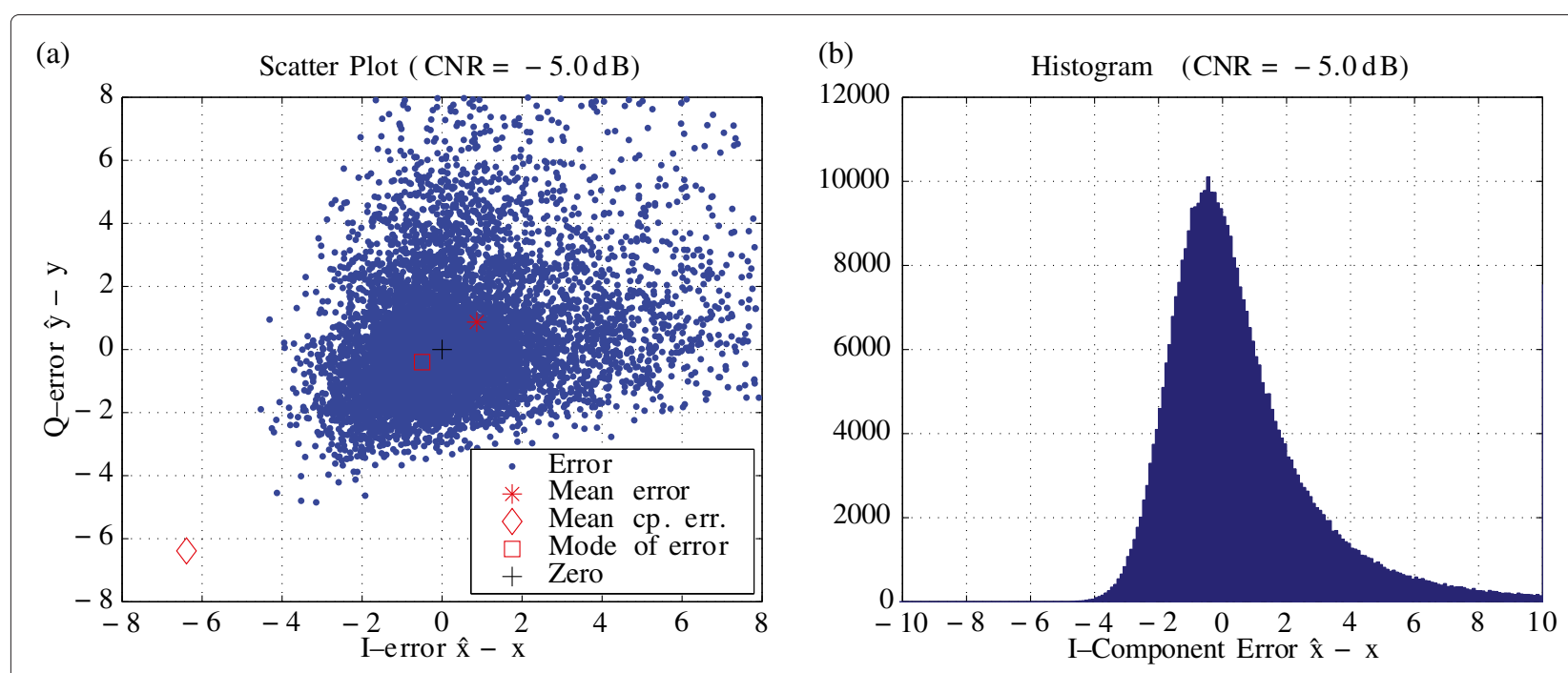

Figure 4 Scatter plot (a) and histogram (b) of estimation error at CNR $=\mathbf{- 5} \mathbf{d B}$. Monte Carlo simulations of the fast algorithm using $4 \times 10^{5}$ realisations. (a) A scatter plot of 8,000 error points and additionally the mean and mode of the error as well as the mean of the error with compensated bias (Mean cp. err.) based on Equation 15. (b) Histogram showing the distribution of the relative error of the / component. 

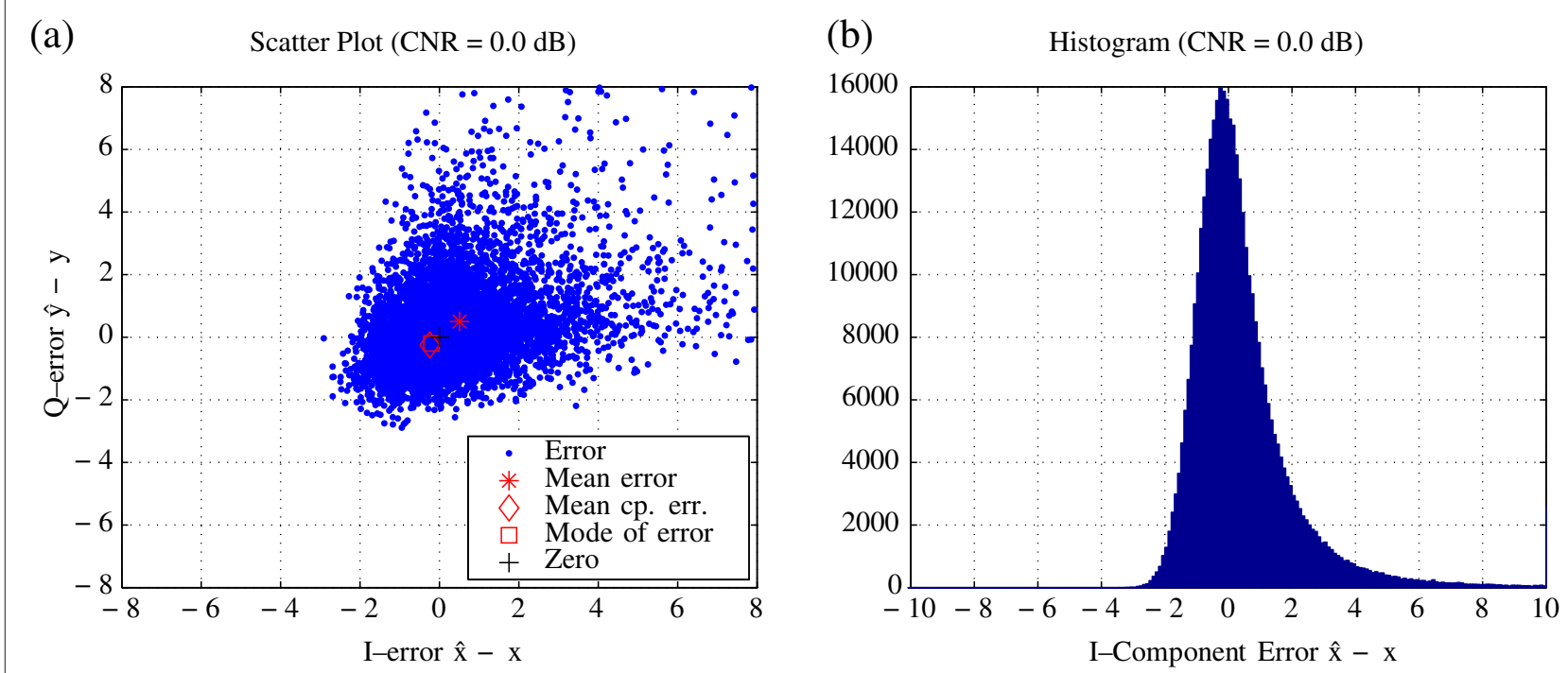

Figure 5 Scatter plot (a) and histogram (b) of estimation error at CNR $=\mathbf{0} \mathbf{d B}$. Monte Carlo simulations of the fast algorithm using $4 \times 10^{5}$ realisations. (a) A scatter plot of 8,000 error points and additionally the mean and mode of the error as well as the mean of the error with compensated bias (Mean cp. err.) based on Equation 15. (b) Histogram showing the distribution of the relative error of the / component.

was controlled via a standard PC. The detailed measurement set-up is described in the next section. Further, we will present the results of the pure gradient algorithm, the fast algorithm and two enhanced algorithms based on these two.

\subsection{Set-up}

Figure 7 illustrates the measurement set-up used throughout the measurements. A photograph of the measurement set-up is shown in Figure 8. The algorithms were evaluated for a pure sine wave carrier signal, which was created in the generator shown in the upper left corner. Its output signal is split up into the upper leakage generating path, and the lower cancellation path, which consists of an LCC module called carrier cancellation unit 1 (CCU1), which is described in detail in [13]. It consists of a vector modulator and an amplifier, which raises the level of the compensation signal to compensate for the coupling losses in (a)

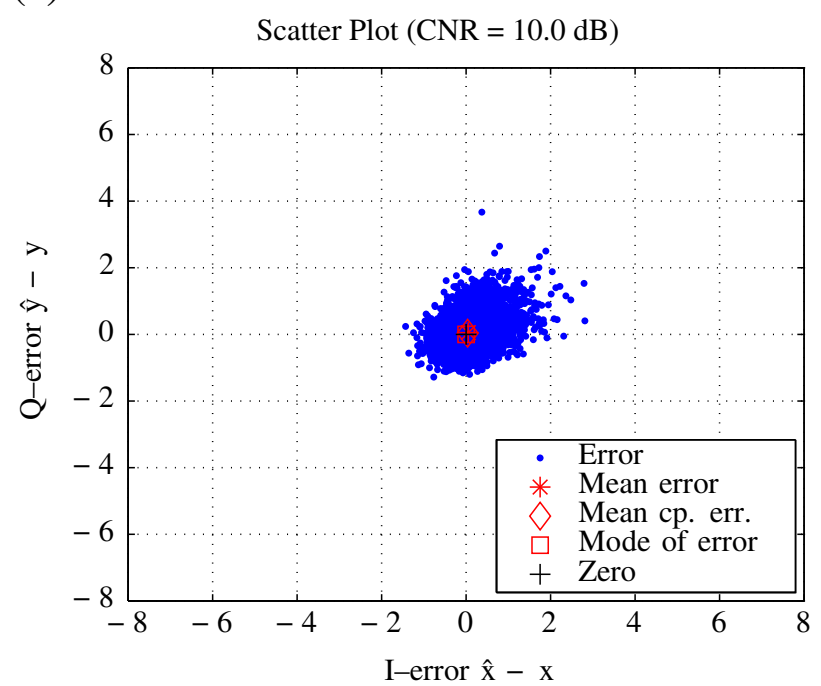

(b)

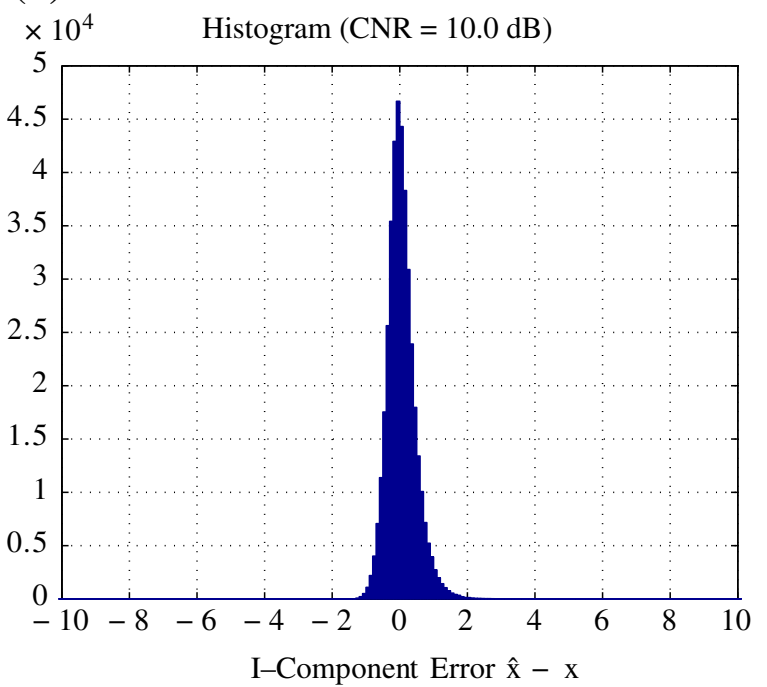

Figure $\mathbf{6}$ Scatter plot (a) and histogram (b) of estimation error at CNR $=\mathbf{1 0} \mathbf{d B}$. Monte Carlo Simulations of the fast algorithm using $4 \times 10^{5}$ realisations. (a) A scatter plot of 8,000 error points and additionally the mean and mode of the error as well as the mean of the error with compensated bias (Mean cp. err.) based on Equation 15. (b) Histogram showing the distribution of the relative error of the / component. 


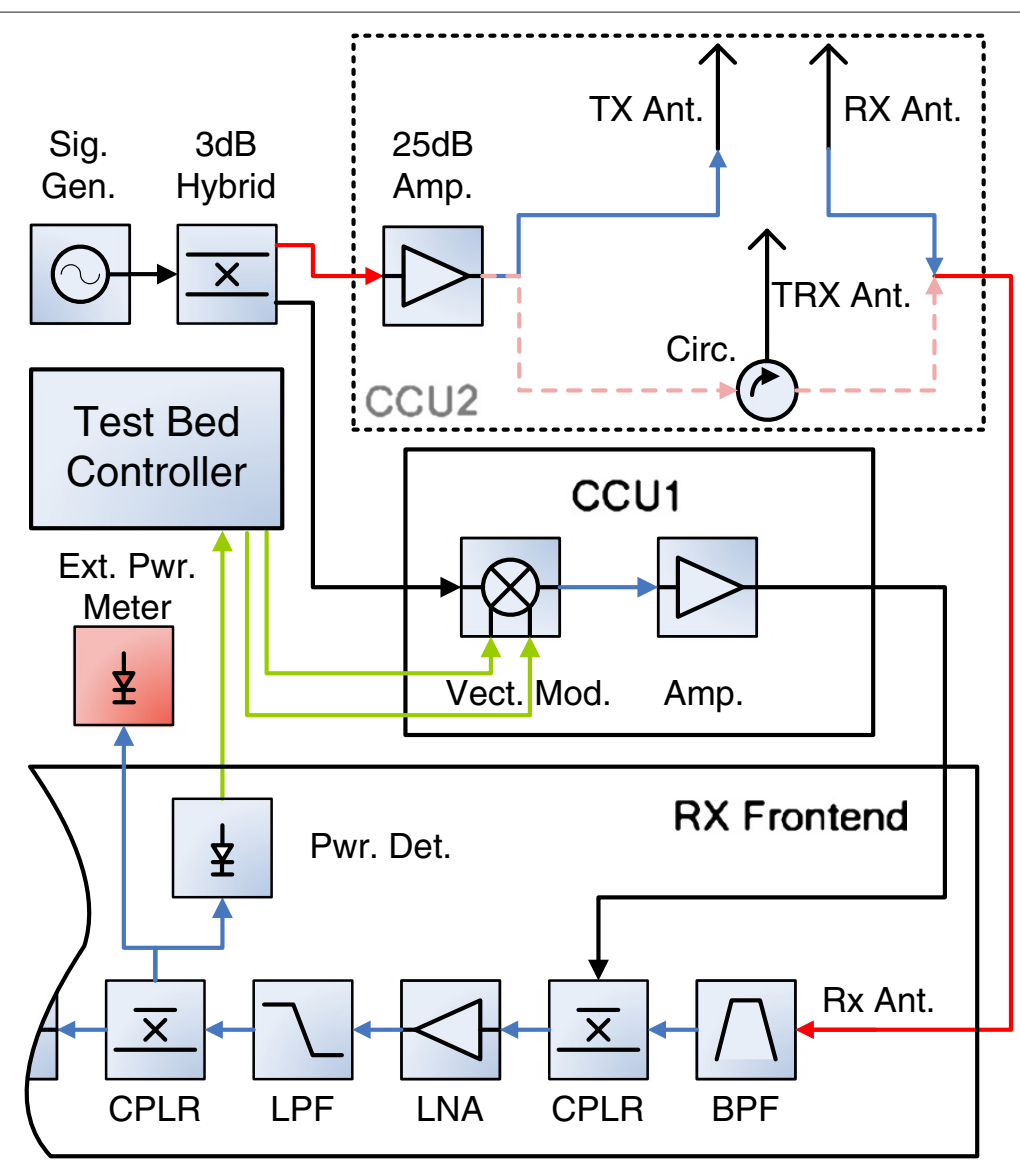

Figure 7 Measurement set-up. This is a block diagram of the measurement set-up that was used to compare the fast algorithm with the gradient algorithm. The lower part of the picture shows the front end of our testbed's RFID receiver, where the first power detector output is fed into the testbed controller (TBC), which commands all hardware necessary for LCC adaption. The LCC consists of a module called carrier cancellation unit 1 (CCU1), which is controlled from the testbed controller. The leakage signal is either generated by one of two antenna configurations or by replacing this complete block with another CCU2 for artificial leakage generation.

the receiver. The upper leakage generating part either consists of one of two antenna scenarios or is replaced by a second CCU to artificially generate a well-defined leakage signal. When using the antennas, we first amplify the signal using an amplifier with a gain of $25 \mathrm{~dB}$ and then either feed a transmit (TX) antenna and pick up the signal with a receive (RX) antenna to mimic a bistatic RFID scenario, or we use a monostatic scenario with a single transceive (TRX) antenna and a circulator. The antennas had a gain of $8 \mathrm{dBi}$, and in the bistatic scenario, they were placed side by side facing the same direction for reasonable TX-RX decoupling.

Both leakage and compensation signals were routed to the front end of our RFID receiver [27] where they combine in the first directional coupler. This summed signal is then fed through a LNA and a LPF and finally reaches the second directional coupler. Here, a part of the received signal is extracted and fed to the internal power detector and an external power metre. The internal logarithmic power detector output is routed to the testbed controller (TBC) where it is used as a source signal for the tested LCC adjustment routines. Except for the LNA chosen for its highly linear operation, it is not affected by nonlinearity of any receiver hardware component and, therefore, is comparable to detector A discussed in Section 2.6. The external power metre was used to obtain measurement data only, but not for use in the adjustment algorithms. The TBC provides 16-bit ADCs to capture the power metre output signals and 12-bit DACs to control the $I / Q$ channels of CCU1 and CCU2, corresponding to 4,096 possible settings for each channel.

The receiver's power detector provides a voltage which is logarithmically dependent on the power detector's input power. Since the fast algorithm demands for a linear amplitude detector, we used a function to convert every voltage measurement from the TBC corresponding to a power measurement from the power detector into a number. This linearising function consists of an exponential 


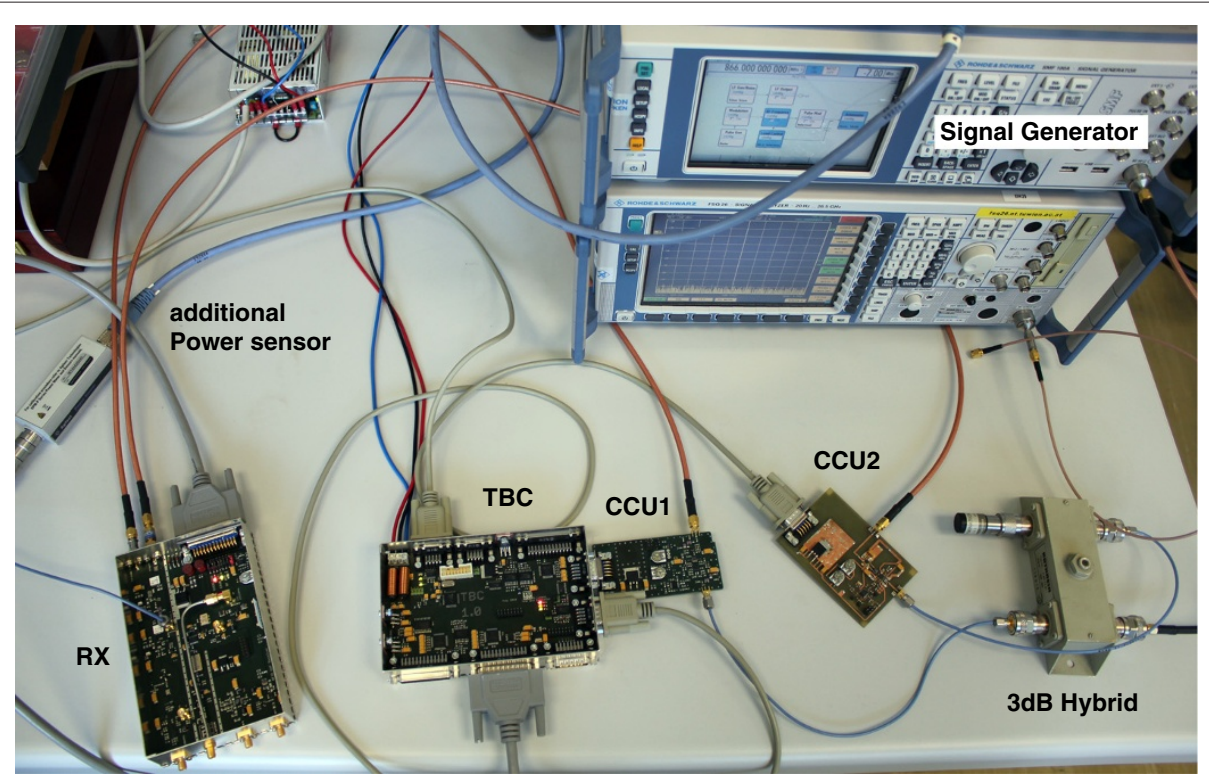

Figure 8 Measurement set-up. This picture shows the measurement set-up that was used for all measurements presented in this paper. Reprinted from Fig. three in [25], with permission from the IEEE.

function and additionally a multiplicative constant. This constant was chosen to result in most linear amplitude readings with respect to the output of the function. The LCC composed of CCU1 was calibrated with respect to these amplitude measurements by disconnecting the red antenna input signal in Figure 7 and by performing separate sweeps in the $I$ and $Q$ domains, always setting the unswept channel to zero. These sweeps were performed with a step size of 10 corresponding to 410 recorded amplitude measurements per channel. The values obtained in these measurements were stored in a lookup table and aided by interpolation enabled to set the LCC to the correct probing values $c_{1}$ and $c_{2}$ necessary for steps two and three of the fast algorithm and finally to $c_{\mathrm{opt}}$ found by Equation 5 .

\subsection{Measurement results}

For a first feasibility test of the hardware and the fast algorithm in a realistic RFID scenario, we conducted measurements using the monostatic and bistatic set-ups. For different orientations of the antennas in the laboratory room, isolation gains of the fast algorithm ranging from 8.1 to $27.9 \mathrm{~dB}$ were reached. Here, we define the TX to $\mathrm{RX}$ isolation gain $G_{\mathrm{I}}$ as the ratio of uncancelled leakage power $P_{\mathrm{L}}$ divided by the remaining power of the cancelled leakage signal $P_{\mathrm{CL}}$ :

$$
G_{\mathrm{I}}=\frac{P_{\mathrm{L}}}{P_{\mathrm{CL}}}=\frac{r^{2}}{|\hat{x}-x|^{2}+|\hat{y}-y|^{2}} .
$$

According to this definition, the isolation gain directly reveals the improvement of the used cancellation algorithm in comparison to a system without LCC usage. When expressed in decibels, the isolation gain ranges from small negative values for badly misadjusted LCCs to large positive values for properly adjusted LCCs.

For comparison, we also implemented a gradient search algorithm according to Section 2.2, where we chose the gradient finding step size to be $\delta=10$ in general and $\delta=2$ for LCC settings close to the optimum LCC setting point. We used a variable step size $\mu$ which was adapted proportional to $\sqrt{\nabla_{n}}$ according to an empirically chosen factor. Both adaption schemes aided to securely find the optimum LCC setting by reducing the step sizes close to the optimum point. The gradient search algorithm reached isolation gains ranging from 14.7 to $24.7 \mathrm{~dB}$.

For a more systematic analysis, we replaced the RFID antenna scenario with a second CCU module to retrieve systematic leakage cancellation measurements. CCU2 was swept over the $I / Q$ space in 65 steps per channel producing 4,225 points in total. Figure 9 shows the resulting isolation gain $G_{\mathrm{I}, \mathrm{F}}$ of the fast algorithm in a pseudo-colour plot. The figure is plotted with respect to the $I / Q$ leakage plane, i.e. the CCU2 DAC control plane. Since 12-bit DACs are used for both the $I$ and $Q$ channels, this plane ranges from $-2,048$ to 2,047 . In the centre, no isolation gain is obtainable since the input power $P_{\mathrm{L}}=0$. For increasing input powers, the isolation gain of the fast algorithm increases as well. Here, the presented gain is also limited by the low end of the power metre's dynamic range, since the absolute power levels at the power metre after the coupler and splitting losses are rather low. We note a decrease in isolation gain in three of the four 


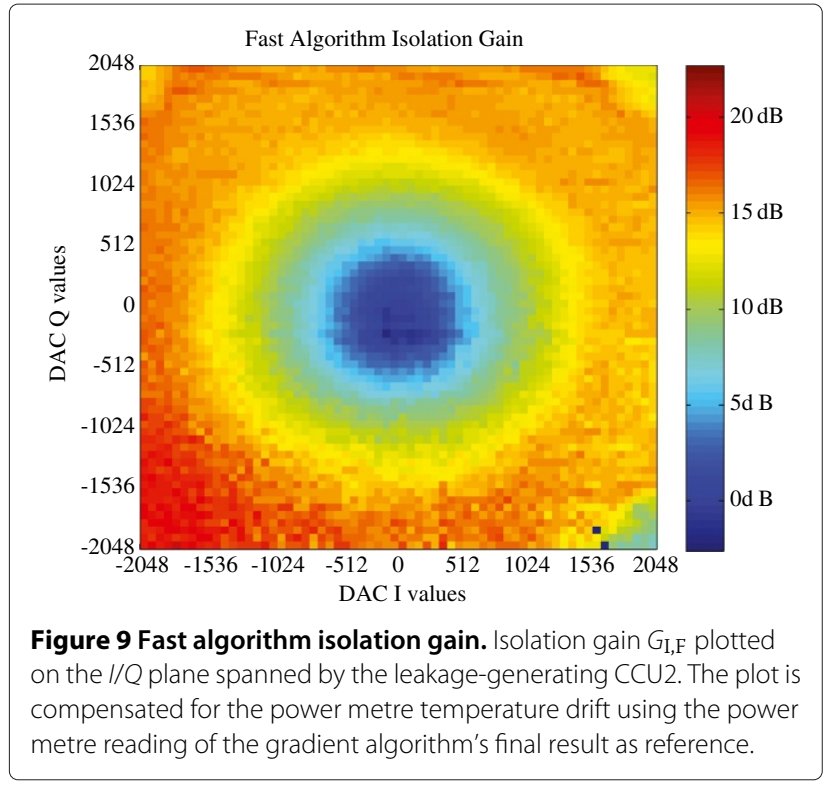

corners for high input leakage powers. This effect is also visible in Figure 10. Here, the ratio of the isolation gains of the gradient algorithm and the fast algorithm is plotted. Since we believe the gradient algorithm converges to the optimum LCC setting point under the given hardware constraints, we may interpret Figure 10 as the fast algorithm's isolation gain deviation from the optimum value. For large areas, the performance of the fast algorithm is very good; in $95 \%$ of the measured points, the error is below $3 \mathrm{~dB}$. We again see the degradation at the same three corners.

For further analysis of this behaviour, we discuss the relative error vector magnitude of the fast algorithm when compared to the gradient algorithm

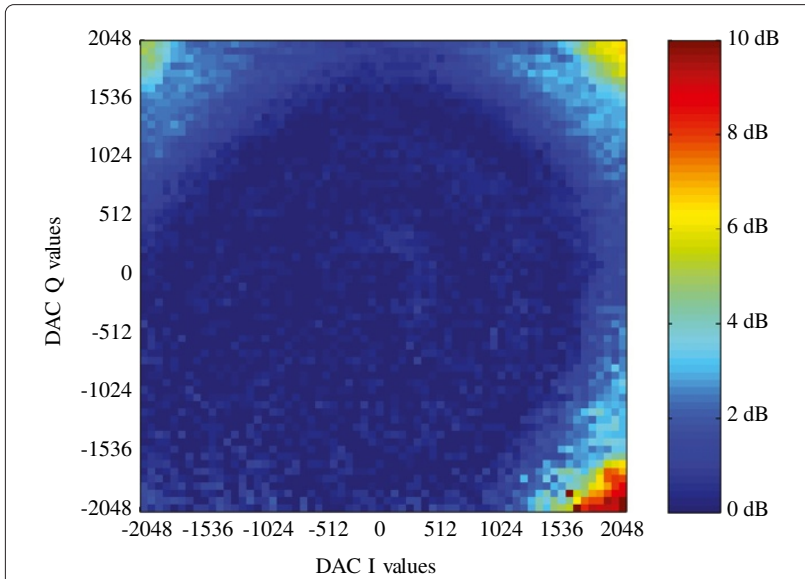

Figure 10 Isolation gain ratio. Isolation gain ratio $\frac{G_{I, G}}{G_{I, F}}$ of the gradient algorithm and the fast algorithm plotted on the I/Q plane spanned by the leakage-generating CCU2 (compare with Fig. seven in [25], with permission from the IEEE).
$\sqrt{\left|\hat{x}-\hat{x}_{\mathrm{G}}\right|^{2}+\left|\hat{y}-\hat{y}_{\mathrm{G}}\right|^{2}} / N$, which is shown in Figure 11 . In contrast to the plots discussed before, we now have drawn the plot with respect to the LCC's $I / Q$ plane, i.e. the CCU1 DAC values. Each plotted point corresponds to the estimated $I$ and $Q$ values $\hat{x}_{\mathrm{G}}$ and $\hat{y}_{\mathrm{G}}$ of the gradient algorithm for this leakage signal. We see that the resulting diagram is a square which is slightly smaller than the LCC's $I / Q$ space and rotated counterclockwise. The good agreement of the shape of the diagram to a perfect square is a sign that the gradient algorithm captures the true leakage values very closely. The rotation is due to the phase shift between leakage and compensation paths, which is mainly caused by the cabling of the measurement set-up. The maximum leakage power was chosen to be slightly smaller than the maximum cancellation power so that all leakage settings can be compensated. The majority of the measured points, $68 \%$, exhibit an error smaller than $3 \%$.

We see that the lower left quadrant exhibits very low error magnitudes, typically below $2 \%$. For all other quadrants, we notice a behaviour which is primarily dependent on the leakage amplitude which results in concentric circles of higher and lower estimation errors of the fast algorithm. For large amplitudes in these quadrants, relative error magnitudes larger than $5 \%$ are observed which corresponds to the degraded performance in the three corners that we noted before. This behaviour is explained by the imperfect amplitude detector. Although the detector is situated almost directly at the receiver input, which is a prerequisite for linear operation under the constraints of reasonable receiver hardware demands (see Section 2.6), we see that the estimation error grows for larger amplitudes. This is due to the fact that we are

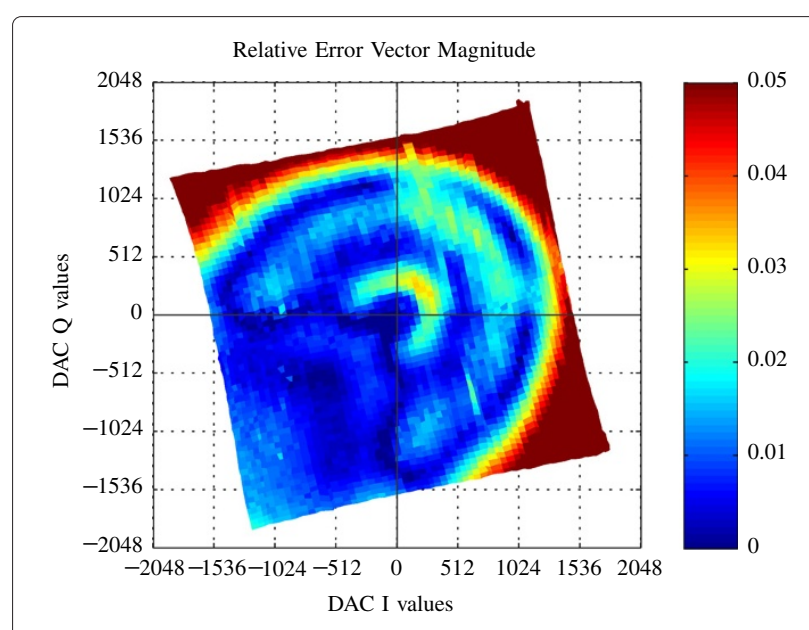

Figure 11 Relative error vector magnitude. Relative magnitude of the setting error of the fast algorithm when compared to the gradient search algorithm $\sqrt{\left|\hat{x}-\hat{x}_{G}\right|^{2}+\left|\hat{y}-\hat{y}_{G}\right|^{2}} / N$, plotted on the I/Q space of CCU1 on the estimated leakage values $\hat{x}_{G}$ and $\hat{y}_{G}$ (compare with Fig. six in [25], with permission from the IEEE). 
using a logarithmical power detector which decreases the sensitivity for high input amplitudes, and the conversion to linear values is not perfect. In the lower left quadrant, this effect is not observed, since here the probing signals of the fast algorithm have opposite polarity to the leakage signals and therefore result in small signal amplitudes for steps two and three of the fast algorithm according to Equations 3 and 4. This theory is fortified when we inspect Figures 12 and 13, which separately show the relative estimation errors of the $I$ and $Q$ components with respect to the gradient algorithm estimate. Figure 12 represents the error of the inphase component, which is very small for leakage signals with a negative $I$ component, and fluctuates for leakage signals with a positive $I$ component until it rapidly rises for large leakage amplitudes. In this domain, the estimator overestimates the $I$ component. The critical step in the fast algorithm is the second measurement where the probing signal is applied and the resulting sum amplitude is measured. In the left quadrants of Figure 12, this sum amplitude is ranging from 0 to $\sqrt{2} r$, where $r$ is the amplitude of the leakage signal. In the right quadrants, the sum amplitude ranges from $\sqrt{2} r$ to $2 r$. The relatively high sum amplitude with a small relative change and the use of the logarithmic detector lead to the worse performance of these right quadrants. The same is true for the upper quadrants of the $Q$ error shown in Figure 13. Combining these two error patterns finally leads to the estimation error we see in Figure 11.

\section{Enhanced procedures}

Based on the practical and theoretical findings, we present two enhancements to the set of algorithms described in Section 2.

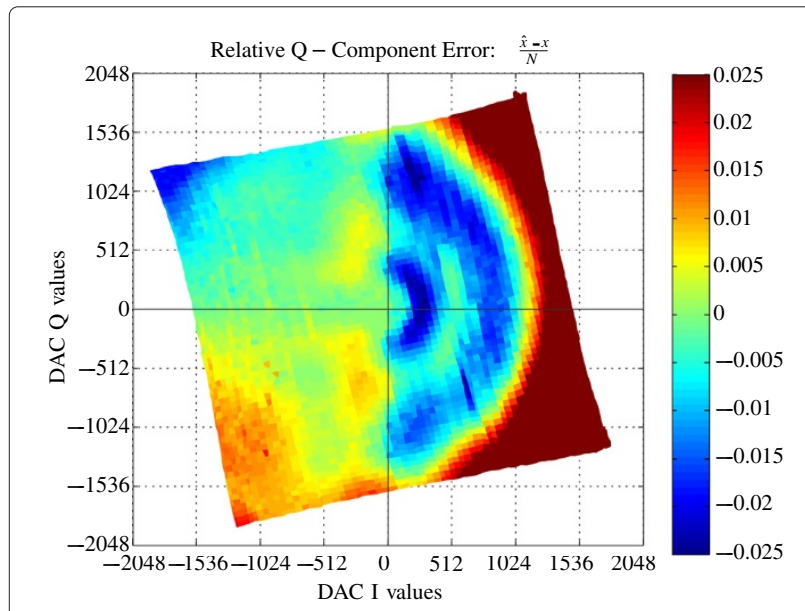

Figure 12 I component error. Relative / component setting error of the fast algorithm $\frac{\hat{x}-\hat{x}_{G}}{N}$, plotted on the I/Q space of CCU1 on the estimated leakage values $\hat{x}_{\mathrm{G}}$ and $\hat{y}_{G}$.

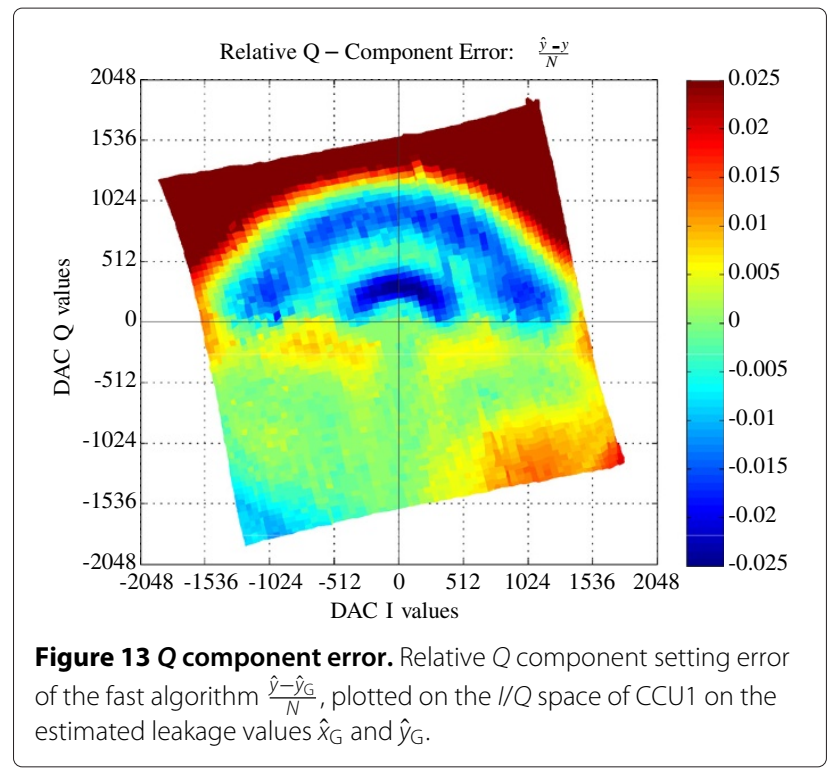

\subsection{Fast algorithm with bipolar probing signals}

We have seen from the measurement results that for our practical system, the fast algorithm is limited by the nonlinear behaviour of the logarithmical power detector. The quadrant which lies in the negative $I$ and $Q$ sectors of the leakage signal is only slightly affected, since here the probing signal and the leakage signal partially cancel each other and result in a low amplitude signal which is measured in the second and third steps of the fast algorithm. We extend this beneficial behaviour to all four $I / Q$ quadrants using positive and negative probing signals for $I$ and $Q$ components. For the negative probing signals, Equation 5 becomes

$$
\begin{aligned}
& \hat{x}=\frac{r_{0}^{2}-\frac{1}{2} r_{1}^{2}}{r_{0}}=x, \\
& \hat{y}=\frac{r_{0}^{2}-\frac{1}{2} r_{2}^{2}}{r_{0}}=y .
\end{aligned}
$$

Since the fast algorithm in the first step measures the leakage amplitude $r$, it is immediately clear after the second step if this step should be repeated with a negative probing signal, because the resulting measured amplitude in the second step is smaller than $\sqrt{2} r$ when the $I$ component lies in the beneficial region. Using this conditional re-measurement technique, the fast algorithm's step count stated in Table 1 is increased from three to three to five, which has an average number of four steps. Of course, it is also possible to always perform all five measurements; then, even averaging of the two estimates of the same $I / Q$ component is possible. This possibly makes sense for leakage signals whose signal power is concentrated in the other $I / Q$ component so that the leakage signal and probing signal are almost orthogonal to each other. 


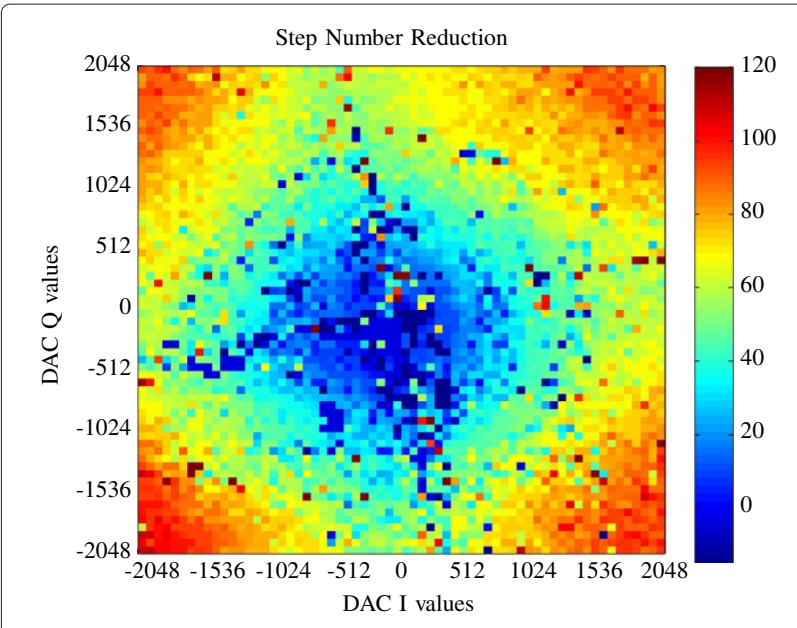

Figure 14 Step reduction. Reduction in number of steps required to converge to final value when comparing the pure gradient search algorithm with a gradient search algorithm aided by a fast algorithm starting point.

When using this technique of bipolar probing signals, the bias derived in Section 3.1 does not change as long as the statistical properties of the noise components $U$ and $V$ are the same; since then, Equation 15 does not depend on $x$ or $y$. The variance of the standardised estimation error for medium to high CNR values for this algorithm is smaller than the standard deviation of the regular fast algorithm, as we see from our Monte Carlo simulations in Figure 3. Therefore, this improvement which we had considered to overcome limitations due to the linearity of the amplitude detector also improves the estimation in the case of noise-limited operation.

Based on the measurements presented in Figure 10, we calculate the average isolation gain ratio of the beneficial quadrant shown as the lower left quadrant in Figure 11 and get $0.48 \mathrm{~dB}$. Thus, we conclude that using the bipolar probing method for the fast algorithm, we reach the isolation gain of the gradient algorithm with a mean penalty of only $0.48 \mathrm{~dB}$.

\subsection{Fast algorithm-aided gradient algorithm}

In practical RFID scenarios, many factors limit the performance of the fast algorithm, which are mainly based on nonlinear detector behaviour and imperfect LCC calibration. However, the fast algorithm may be used to aid the gradient algorithm by setting the starting point of the iterative search to the values found by the fast algorithm. We compare this fast algorithm-aided gradient algorithm with our standard gradient algorithm using the same measurement set-up as described in Section 4.1. Using the monostatic and bistatic antenna scenarios, we compare the necessary number of steps for the algorithms to converge. If we stick to our step definition as stated in
Section 2.6, the pure gradient algorithm required 39 to 135 steps, while the aided algorithm for the same scenarios was content with 21 to 60 steps.

We repeated the measurements with the systematic leakage sweep generated by CCU2 to compare the two algorithms on the complete $I / Q$ plane. A graphical representation of the results is given in Figure 14, which shows the reduction in number of steps when compared to the gradient algorithm starting at zero. We see that close to zero, no improvement is possible since the starting point of the pure gradient search algorithm is zero anyway. For larger leakage amplitudes, which are practically more relevant, the fast algorithm provides a better starting point, and reductions up to 120 steps are possible. On average, the pure gradient algorithm requires 75 steps while the aided algorithm only demands 21 steps, which is a decrease by $72 \%$.

\section{Conclusions}

This contribution discussed the performance of wellknown algorithms and novel enhanced procedures for the automatic adjustment of leakage carrier cancellers in RFID readers. We briefly described three well-known algorithms and the fast algorithm published in [25] and discussed their requirements on receiver hardware and performance in terms of their step count. Further, we carried out an analytical noise analysis for the fast adjustment algorithm and derived its bias behaviour. The presented analytic approximation of the estimator bias holds for the high CNR regime where the bias is very small and can be safely ignored. The analytical results were compared to numerical simulation studies which were used to show the estimator's error distribution for varying CNR levels. We found that the bias at low CNR values is negligible in favour of a mode of the error which is close to zero. Thus, it was shown that for both high and low CNR regions, bias compensation of the fast algorithm is not an issue.

We demonstrated the practical performance of the fast adjustment algorithm using our unmodified RFID reader hardware platform. Although this hardware only implements a logarithmic power detector, a systematic scan of the leakage $I / Q$ plane demonstrated that $95 \%$ of the measurements show a maximum penalty in isolation gain of $3 \mathrm{~dB}$ when compared to the much slower gradient search algorithm. We further proposed and discussed two enhanced procedures based on the fast algorithm. The first procedure is an extension and uses bipolar probing signals, which enables accurate LCC setting with a mean error of $0.48 \mathrm{~dB}$, while the average step count is only increased to four. The second procedure is an enhancement to the gradient search algorithm by setting an improved starting point. This reduces the average step count by $72 \%$. 


\section{Competing interests}

The authors declare that they have no competing interests.

\section{Acknowledgements}

This work has been funded by the Christian Doppler Laboratory for Wireless Technologies for Sustainable Mobility. The financial support by the Federal Ministry of Economy, Family and Youth and the National Foundation for Research, Technology and Development is gratefully acknowledged. We would further like to thank our colleague Robert Dallinger for the many inspiring and valuable discussions.

\section{Author details}

${ }^{1}$ Institute of Telecommunication, Vienna University of Technology, 1040,Vienna, Austria. ${ }^{2}$ Christian Doppler Laboratory for Wireless Technologies for Sustainable Mobility, Vienna University of Technology, 1040, Vienna, Austria.

Received: 31 December 2012 Accepted: 21 March 2013

Published: 7 May 2013

\section{References}

1. R Want, An introduction to RFID technology. IEEE Pervasive Comput. 5, 25-33 (2006)

2. H Stockman, Communication by means of reflected power. Proc. IRE. 36(10), 1196-1204 (1948)

3. PV Nikitin, KVS Rao, R Martinez, Differential RCS of RFID tag. Electron. Lett. 43(8), 431-432 (2007)

4. PV Nikitin, KVS Rao, Theory and measurement of backscattering from RFID tags. IEEE Antennas Propag. Mag. 48(6), 212-218 (2006)

5. DM Dokin, The RF in RFID, 1st edn. (Newnes/Elsevier, Burlington, 2008)

6. JP Curty, M Declercq, C Dehollain, N Joehl, Design and Optimization of Passive UHF RFID Systems, 1st edn. (Springer, New York, 2007)

7. K Penttilä, L Sydänheimo, M Kivikoski, Implementation of Tx/Rx isolation in an RFID reader. Int. J. Radio Freq Identification Technol Appl. 1, 74-89 (2006)

8. Y Liu, Q Zhang, M Zheng. Signal analysis and design criteria for UHF RFID reader. ITS Telecommunications Proceedings, Chengdu, June 2006 (IEEE, Piscataway, 2006), pp. 233-236

9. TI Al-Mahdawi, Adaptive coherent RFID reader carrier cancellation, U.S patent US. 2006/183454. 17 Aug 2006

10. DP Villame, JS Marciano Jr. Carrier suppression locked loop mechanism for UHF RFID readers. IEEE International Conference on RFID, Orlando, April 2010 (IEEE, Piscataway, 2010), pp. 141-145

11. R Langwieser, $G$ Lasser, C Angerer, M Fischer, AL Scholtz. Active carrier compensation for a multi-antenna RFID reader frontend. 2010 IEEE MTT-S International Microwave Symposium Digest, Anaheim, May 2010, (IEEE, Piscataway, 2010), pp. 1532-1535

12. I Mayordomo, J Bernhard. Implementation of an adaptive leakage cancellation control for passive UHF RFID readers. IEEE International Conference on RFID, Orlando, April 2011 (IEEE, Piscataway, 2011), pp. 121-127

13. G Lasser, R Langwieser, AL Scholtz. Broadband suppression properties of active leaking carrier cancellers. IEEE International Conference on RFID, Orlando, April 2009 (IEEE, Piscataway, 2009)

14. P Pursula, M Kiviranta, H Seppä, UHF RFID reader with reflected power canceller. IEEE Microw. Wireless Compon, Lett. 19, 48-50 (2009)

15. T Xiong, X Tan, J Xi, X Min, High TX-to-RX isolation in UHF RFID using narrowband leaking carrier canceller. IEEE Microw. Wireless Compon, Lett. 20(2), 124-126 (2010)

16. LW Mayer, AL Scholtz. Circularly polarized patch antenna with high Tx / Rx-separation. IEEE International Conference on RFID, Orlando, April 2009 (IEEE, Piscataway, 2009), pp. 213-216

17. JY Wang, B LV, WZ Cui, W Ma, JT Huangfu, LX Ran, in Progress in Electromagnetics Research Symposium. Isolation enhancement based on adaptive leakage cancellation, pp. 1059-1063. Xi'an, 22-26 March 2010

18. Inc. Impinj. Indy @ R2000 Reader Chip (IPJ-R2000). REV, 1.3 2012, Impinj, Inc., Seattle

19. J Lee, j Choi, KH Lee, B Kim, M Jeong, Y Cho, H Yoo, K Yang, S Kim, SM Moon, JY Lee, S Park, W Kong, J Kim, TJ Lee, BE Kim, BK Ko. A UHF mobile RFID reader IC with self-leakage canceller. IEEE Radio Frequency Integrated Circuits RFIC Symposium, Honolulu, June 2007 (IEEE, Piscataway, 2007), pp. 273-276
20. P Beasley, A Stove, B Reits, B As. Solving the problems of a single antenna frequency modulated CW radar. Record of the IEEE 1990 International Radar Conference, Arlington, May 1990 (IEEE, Piscataway, 1990), pp. 391-395

21. K Lin, YE Wang, CK Pao, YC Shih, A Ka-Band FMCW radar front-end with adaptive leakage cancellation. IEEE Trans. Microw. Theory Tech. 54(12), 4041-4048 (2006)

22. R Langwieser, G Lasser. Measurement and simulation of crosstalk and crosstalk compensation in UHF RFID. Fourth International EURASIP Workshop on RFID Technology, Torino, September 2012 (IEEE, Piscataway, 2012)

23. G Lasser, R Langwieser, R Dallinger, CF Mecklenbräuker. Broadband leaking carrier cancellation for RFID systems. IEEE MTT-S International Microwave Symposium, Montreal, June 2012 (IEEE, Piscataway, 2012)

24. S Haykin, Adaptive Filter Theory, 1st edn. (Prentice-Hall, Upper Saddle River, 1986)

25. G Lasser, W Gartner, R Langwieser, CF Mecklenbräuker, in Fourth International EURASIP Workshop on RFID Technology. Fast algorithm for leaking carrier canceller adjustment. Torino, 27-28 September 2012

26. IA Glover, PM Grant, Digital Communications, 2nd edn. (Pearson, Edinburgh, 2004)

27. R Langwieser, G Lasser, C Angerer, M Rupp, AL Scholtz, in The 2nd International EURASIP Workshop on RFID Technology. A modular UHF reader frontend for a flexible RFID testbed. Budapest, 7-8 July 2008

doi:10.1186/1687-3963-2013-8

Cite this article as: Lasser et al:: Automatic leaking carrier canceller adjustment techniques. EURASIP Journal on Embedded Systems 2013 2013:8.

\section{Submit your manuscript to a SpringerOpen ${ }^{\circ}$ journal and benefit from:}

- Convenient online submission

- Rigorous peer review

- Immediate publication on acceptance

- Open access: articles freely available online

- High visibility within the field

- Retaining the copyright to your article

Submit your next manuscript at $>$ springeropen.com 